\title{
Single-sideband hybrid opto-electronic oscillator
}

\author{
Aurélien Thorette ${ }^{1}$, Marco Romanelli ${ }^{1}$, Steve Bouhier ${ }^{1}$, Frédéric van Dijk $^{2}$, M. Alouini ${ }^{1}$, Marc Vallet ${ }^{1}$ \\ 1. Univ Rennes, CNRS, Institut FOTON - UMR 6082, F-35000 Rennes, France \\ 2. III-V Lab, a joint lab of Thales Research and Technology, Nokia Bell Labs France and CEA LETI, Palaiseau, France
}

Opto-electronic oscillators (OEO) are important building blocks in microwave photonics systems, allowing to obtain low-phase noise optically-carried radiofrequency signals. We have recently demonstrated an OEO scheme based on a monolithic dual-frequency source [1], whose beatnote is self-stabilized using frequency-shifted optical feedback, and a delay line [2]. The main interest of such a setup is that it allows the generation of single-sideband signals, which are thus insensitive to dispersion fading when propagating in optical fibers. The scheme presented in [2] offered good short-term performances. However, it could be affected by mode-hopping due to both the resonant modes of the optical feedback loop, and to those of the optoelectronic loop including the delay line. In the present work, we present significant improvements, namely the complete suppression of mode-hopping, and a significant reduction of the spurs in the phase noise spectrum. This has been obtained by (i) the introduction of a RF interferometer thanks to the use of two delay lines, and (ii) a significant shortening of the optical feedback loop (see Fig. 1(a)) [3].
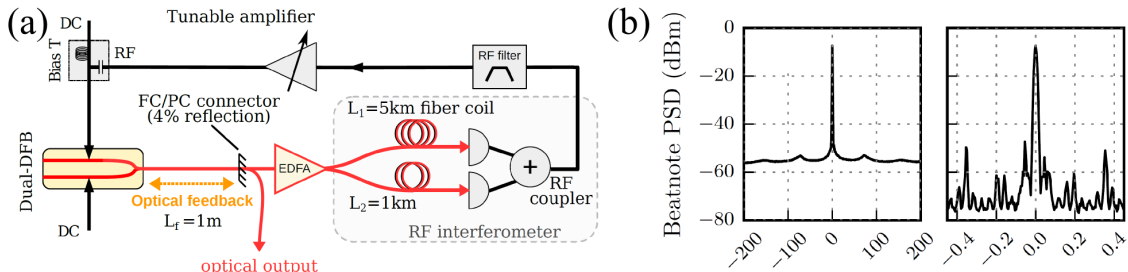

(c)

(d)
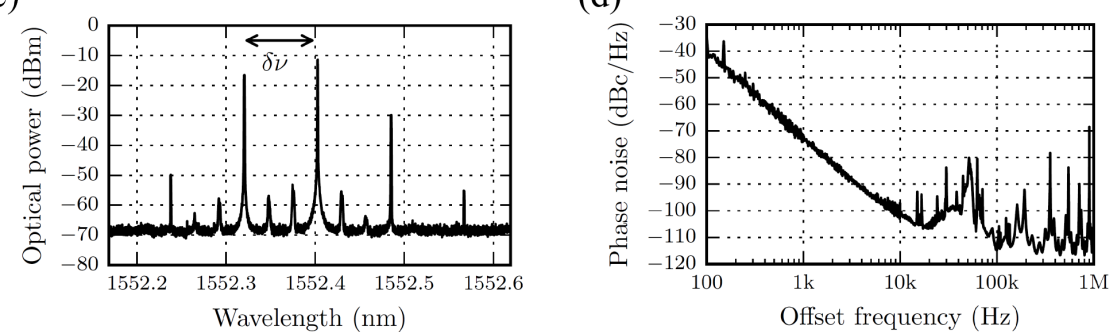

Fig. 1 (a) Setup. The optical feedback is provided by the residual reflection from a fiber connector. One of the two lasers is gain-modulated at the delayed beating frequency by means of a photodiode followed by a $10 \mathrm{GHz}$ RF filter, a RF amplifier and a bias T. (b) Experimental RF spectrum with two different spans. Left: RBW $=1 \mathrm{MHz}$, Right: RBW $=10 \mathrm{kHz}$. (c) Output optical spectrum. The output signal is the beatnote at frequency difference $\delta v=10 \mathrm{GHz}$. (d) Measured phase noise of the RF beatnote.

The RF interferometer leads to suppression, by destructive interference, of the spurs that are due to the fiber loops before the photodiodes. As can be seen from the RF spectrum (right panel of Fig. 1(b)), the spurs close to the carrier are $40 \mathrm{~dB}$ below the main peak. The left panel of Fig. 1(b) shows the RF spectrum on a larger span. The short $(\sim 1 \mathrm{~m})$ optical feedback loop results in broadly spaced external cavity modes, that are efficiently suppressed using a standard RF filter (on the contrary, longer loops require custom filters with high quality factors [2]). As a result, mode hops are completely absent. Note that, in order to reduce the optical feedback loop, we have been obliged to resort to direct current modulation. One may argue that this spoils the single-sideband character of the signal, but the optical spectrum (Fig. 1(c)) demonstrates that it is not so. Indeed, the third optical frequency seen in the spectrum is $15 \mathrm{~dB}$ below the main frequencies; a straightforward calculation shows that, in the worst case, this can reduce the ampitude of the RF signal by only $1.6 \mathrm{~dB}$. So the source is, in practice, almost ideally single-sideband. Finally, good phase noise performances are also obtained (Fig. 1 (d)). Potential phase noise improvement and integration scheme in a single component can be envisioned.

\section{References}

[1] F. van Dijk, A. Accard, A. Enard, O. Drisse, D. Make, and F. Lelarge, "Monolithic dual wavelength DFB lasers for narrow linewidth heterodyne beat-note generation," in Microwave Photonics, 2011 International Topical Meeting on \& Microwave Photonics Conference, 2011 Asia-Pacific, MWP/APMP. IEEE, 2011, pp. 73-76.

[2] M. Vallet, M.Romanelli, G. Loas, F. van Dijk, and M. Alouini, "Self-Stabilized Optoelectronic Oscillator Using Frequency-Shifted Feedback and a Delay Line," IEEE Photon. Technol. Lett. 28, 1088 (2016).

[3] A. Thorette, M. Romanelli, S. Bouhier, F. Van Dijk, M. Vallet, and M. Alouini, "Hybrid opto-electronic oscillator for single-sideband microwave photonics," Elec. Lett. 54, 706 (2018). 